\title{
Laparoscopic repair of femoral hernia
}

\author{
Xue-Fei Yang ${ }^{1}$, Jia-Lin Liu ${ }^{2}$ \\ ${ }^{1}$ Department of Surgery, the University of Hong Kong-Shenzhen Hospital, the University of Hong Kong, Shenzhen 518053, China; ${ }^{2}$ Department of \\ Hepatobiliary Surgery, Shenzhen People's Hospital \& Second Clinical Medical College of Jinan University, Shenzhen 518020, China \\ Correspondence to: Jia-Lin Liu. Department of Hepatobiliary Surgery, Shenzhen People's Hospital, No. 1017, North Dongmen Road, Luohu District, \\ Shenzhen 518020, China. Email: szliujialin@126.com or 4683755@qq.com.
}

\begin{abstract}
Laparoscopic repair of inguinal hernia is mini-invasive and has confirmed effects. Femoral hernia could be repaired through the laparoscopic procedures for inguinal hernia. These procedures have clear anatomic view in the operation and preoperatively undiagnosed femoral hernia could be confirmed and treated. Lower recurrence ratio was reported in laparoscopic procedures compared with open procedures for repair of femoral hernia. The technical details of laparoscopic repair of femoral hernia, especially the differences to laparoscopic repair of inguinal hernia are discussed in this article.
\end{abstract}

Keywords: Femoral hernia; laparoscopic repair; mesh

Submitted Aug 23, 2016. Accepted for publication Sep 01, 2016.

doi: 10.21037/atm.2016.09.31

View this article at: http://dx.doi.org/10.21037/atm.2016.09.31

\section{Introduction}

Femoral hernias account for approximately 3\% to $5 \%$ of abdominal hernias, second only to inguinal hernias. Laparoscopic repair of inguinal hernia is mini-invasive and has confirmed effects (1-3). From the viewpoint of the myopectineal orifice concept and laparoscopic repair, the pathogenesis and treatment principles of femoral and inguinal hernias are basically the same. Femoral hernia could be repaired through the laparoscopic procedures for inguinal hernia. Furthermore, femoral hernias are often associated with inguinal hernias (Figure 1) and are easily missed. Laparoscopic procedures have clear anatomic view in the operation and preoperatively undiagnosed femoral hernia could be confirmed and treated (4). Lower recurrence ratio was reported in laparoscopic procedures compared with open procedures for repair of femoral hernia (5) though undifferentiated quality of life was reported (6).

\section{Surgical options}

Intraperitoneal onlay mesh (IPOM), transabdominal preperitoneal (TAPP) repair, or total preperitoneal (TEP) repair can be chosen.

\section{Preoperative evaluation}

In general, performing color Doppler ultrasonography in the bilateral inguinal region for the identification of hydrocele, great saphenous vein varicosities, and the roots of thigh lipomas as well as enlarged lymph nodes should be emphasized.

\section{Anesthesia}

Endotracheal intubation and general anesthesia are recommended.

\section{Surgical preparation}

In addition to the conventional emphasized preparation, bipolar coagulation should be prepared to stop bleeding during artery rupture around the pubic bone or the femoral ring.

\section{Body position}

Supine position, the same position utilized in laparoscopic inguinal hernia repair. Generally, the chief surgeon stands on the opposite side of the femoral hernia. 


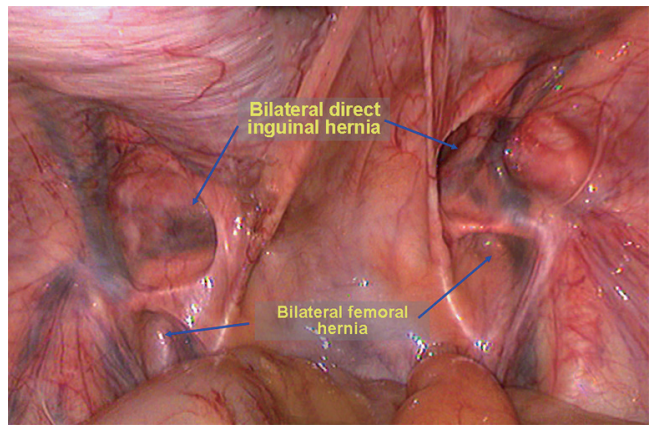

Figure 1 Bilateral femoral hernia associated with bilateral direct hernia.

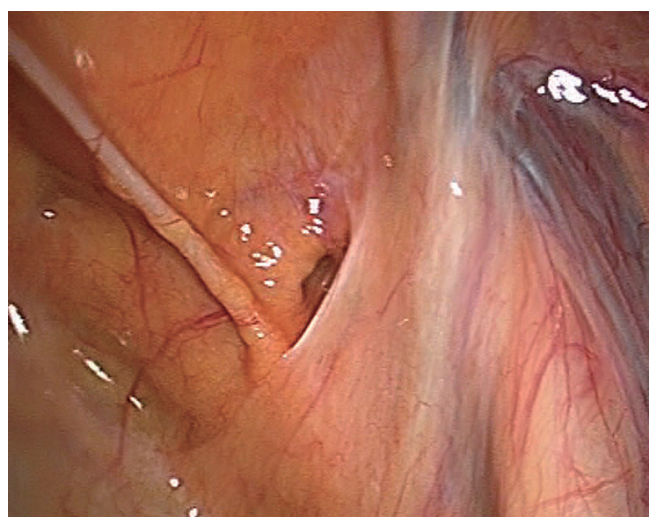

Figure 2 Abdominal exploration confirmed a right femoral hernia.

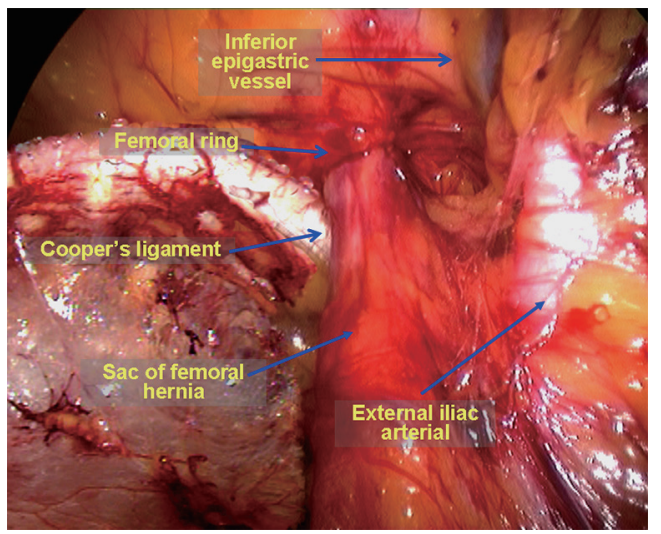

Figure 3 The right side femoral hernia sac can be seen after separating the Retzius space.

\section{Trocar placement}

The same sites utilized in laparoscopic inguinal hernia repair should be used. For TEP repair, one periumbilical 10-mm trocar and two $5-\mathrm{mm}$ midline trocars (in a point of trisection of the connecting line between the umbilicus and the pubic symphysis) are used. The three trocars are in line with each other. For IPOM and TAPP repairs, a $10-\mathrm{mm}$ trocar is placed below the umbilicus and two 5 -mm trocars are placed at the lateral edge of the rectus abdominis at the umbilical level on each side of the abdomen.

\section{Surgical procedures}

Surgical drapes should be conventionally disinfected; pneumoperitoneum should be established; a $10 \mathrm{~mm}$ trocar and a $30^{\circ}$ laparoscope should be placed into the abdominal cavity for examination. It should be noted whether an omentum is present inside the external iliac artery entering into the femoral ring and whether continuous peritoneum disruption at the femoral ring is present or the peritoneum recesses on the inferior side. Occasionally, in clinical practice, patients who are admitted for an inguinal hernia are confirmed to have a femoral hernia by laparoscopy (Figure 2).

Using TEP repair of right femoral hernias in females as an example, after entering the Retzius space and with slight separation, a large amount of tissue that parallels the iliac artery can be observed on the affected side, which crosses the right side of the Cooper ligament and enters the right femoral ring (Figure 3). The right side of the Bogrus space should be initially separated to further separate the hernia sac. A separation clamp can be used to grasp the hernia sac and pull it into the cephalad location; another separation clamp can be used to separate the adhesions to the hernia sac above the femoral ring. Occasionally, the hernia sac has very dense adhesions to the femoral ring and requires meticulous separation with an electric hook. Simultaneously, the assistant can press the femoral ring site, which can help to gradually remove the hernia sac and the closely associated extraperitoneal cellulite from the femoral ring. If repeated attempts to reduce the content (usually omentum clumps) in the hernia sac and the hernia fail, portions of the lacuna ligaments inside the femoral ring can be cut, releasing compression on the femoral ring. Then, the omentum, appendices epiploicae and other involved tissues can be reduced from the femoral canal, achieving reduction of the abdominal cavity (Figure 4). It is necessary to avoid excessive separation outside of the femoral ring to prevent damaging femoral vessels and Corona Mortis vessels. The hernia sac and peritoneum should be folded back to free the cephalad, completely expose the Cooper ligament and avoid hernia sac residue and recurrence. 

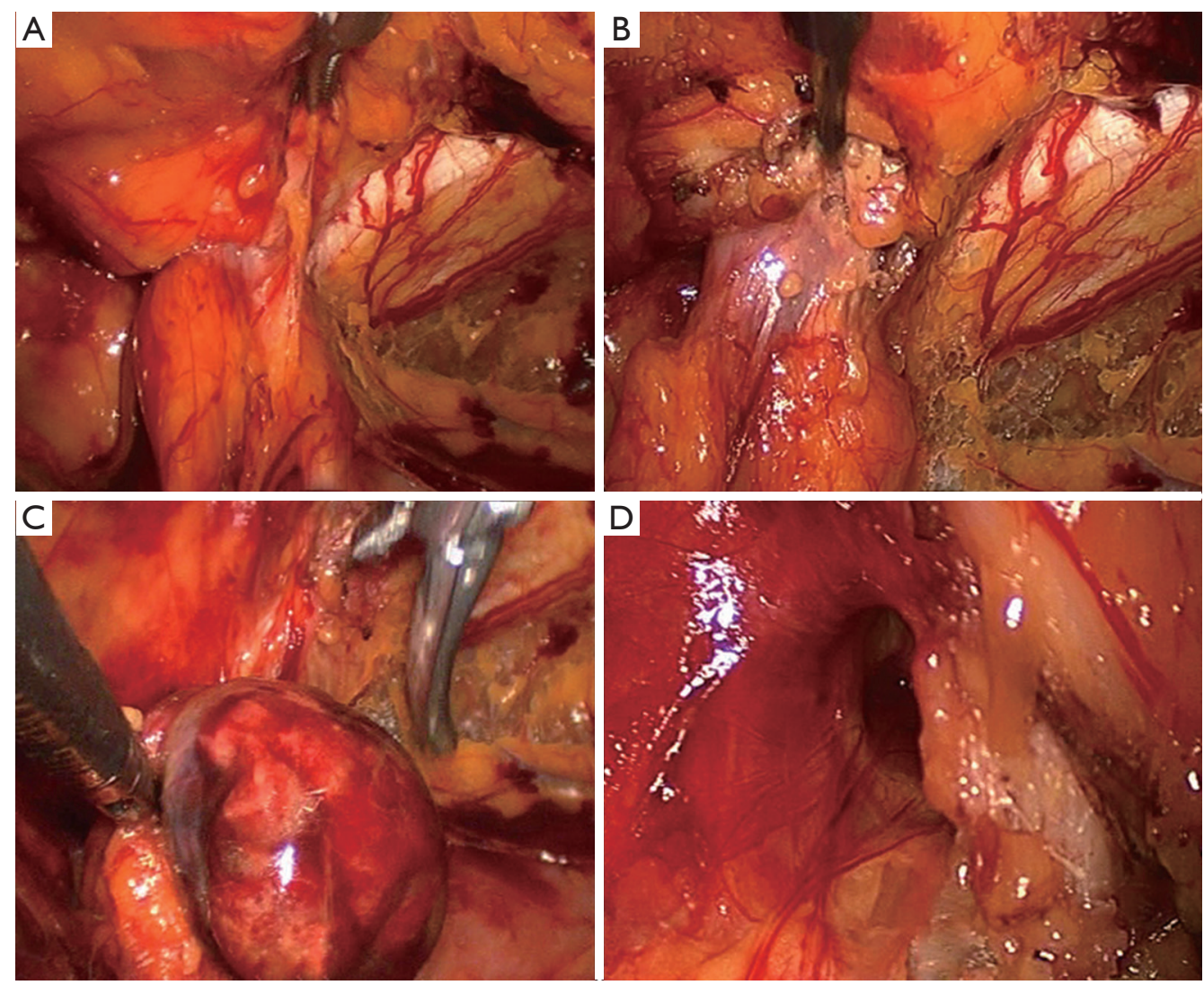

Figure 4 The lacuna ligaments involved in the femoral hernia are cut. (A) Left incarcerated femoral hernia; (B) the left side of lacuna ligaments is cut; (C) reduction of the involved appendices epiploicae; (D) the left femoral ring is exposed.

Polypropylene hernia patches should be cropped during TAPP repair, and after being folded, the patches should be placed in the extraperitoneal space through the umbilical opening cannula. Two separation clamps should be used to spread and smooth the patches, covering the muscular pubic hole. Note that the inner lower edge covers approximately $1 \mathrm{~cm}$ beyond the Cooper ligament. A screw nail should be placed on the Cooper ligament below the femoral ring while trying to avoid Corona Mortis vessels. Screw nails should then be placed at the pubic tubercle, joint tendons, dorsal rectus abdominis and lower right abdominal wall on the affected side. The decision to place a drainage tube depends on the intraoperative drainage situation, similar to laparoscopic inguinal hernia repair. The extraperitoneal drainage, which is placed to avoid hematocele and seroma around the femoral ring, can be removed after 1 day.

\section{Acknowledgements}

Funding: Shenzhen government funding for scientific and technical research and development (JCYJ20140414092023238).

\section{Footnote}

Conflicts of Interest: The authors have no conflicts of interest to declare.

Informed Consent: Written informed consent was obtained from the patient for publication of this manuscript and any accompanying images.

\section{References}

1. Pahwa HS, Kumar A, Agarwal P, et al. Current trends in laparoscopic groin hernia repair: A review. World J Clin Cases 2015;3:789-92.

2. Pisanu A, Podda M, Saba A, et al. Meta-analysis and review of prospective randomized trials comparing laparoscopic and Lichtenstein techniques in recurrent inguinal hernia repair. Hernia 2015;19:355-66. 
3. Li J, Ji Z, Li Y. Comparison of laparoscopic versus open procedure in the treatment of recurrent inguinal hernia: a meta-analysis of the results. Am J Surg 2014;207:602-12.

4. Henriksen NA, Thorup J, Jorgensen LN. Unsuspected femoral hernia in patients with a preoperative diagnosis of recurrent inguinal hernia. Hernia 2012;16:381-5.

5. Andresen K, Bisgaard T, Kehlet H, et al. Reoperation

Cite this article as: Yang XF, Liu JL. Laparoscopic repair of femoral hernia. Ann Transl Med 2016;4(19):371. doi: 10.21037/ atm.2016.09.31 rates for laparoscopic vs open repair of femoral hernias in Denmark: a nationwide analysis. JAMA Surg 2014;149:853-7.

6. Cox TC, Huntington CR, Blair LJ, et al. Quality of life and outcomes for femoral hernia repair: does laparoscopy have an advantage? Hernia 2016. [Epub ahead of print]. 